\title{
Effects of Podocyte Injury on Glomerular Development
}

\author{
JI MA, MICHELE ROSSINI, HAI-CHUN YANG, YIQIN ZUO, AGNES B. FOGO, AND IEKUNI ICHIKAWA
}

\author{
Division of Pediatric Nephrology [J.M., Y.Z., A.B.F., I.I.], Department of Pathology [M.R., H.-C.Y., A.B.F.], Vanderbilt University Medical \\ Center, Nashville, TN 37232; Division of Nephrology [J.M., H.-C.Y.], Hua Shan Hospital, Fudan University, Shanghai 200040, China; \\ Department of Pediatrics [I.I.], Tokai University School of Medicine, Isehara, 259-1193, Japan
}

\begin{abstract}
To study the effects of podocyte injury on glomerular maturation and underlying mechanisms of such effects, puromycin aminonucleoside (PAN) was given to neonatal mice at $1 \mathrm{~d}$ post partum (1 dpp). Mice with PAN injection had smaller kidney weight (KW) and body weight (BW) at all times and smaller KW/BW at 4, 8 , and 12 dpp versus normal saline (NS) controls. Electron microscopy (EM) revealed nearly complete podocyte foot process effacement and segmental microvillous transformation as early as $2 \mathrm{dpp}$, preceding proteinuria. PAN-injected kidneys showed significantly fewer glomerular capillary loops and decreased glomerular maturation index, as well as less CD31+ endothelium in cortical glomeruli at $12 \mathrm{dpp}$ versus NS controls. Glomerular mesangial injury and glomerulosclerosis along with proteinuria were noted in PANinjected kidneys starting from $30 \mathrm{dpp}$. Systolic blood pressure was increased significantly by $60 \mathrm{dpp}$ in PAN mice. PAN mice also had significantly decreased Flk-1 and Tie2 mRNA expression and increased angiopoitein-1 (Ang-1) expression, without change in vascular endothelial growth factor (VEGF) at 2 dpp versus NS. Our study shows that podocyte injury in neonatal mice kidneys alters the expression of key capillary growth modulators in glomeruli, leading to abnormal development of glomerular capillaries, with subsequent development of proteinuria, hypertension, and glomerulosclerosis. (Pediatr Res 62: 417-421, 2007)
\end{abstract}

$\mathrm{T}$ he glomerular filtration barrier is composed of podocytes, endothelial cells, and the glomerular basement membrane (GBM). Any structural changes in these three components can result in alterations in glomerular function (1). Some growth factors promoting endothelial proliferation, elongation, and migration, such as vascular endothelial growth factor (VEGF) and angiopoietin-1 (Ang-1), are primarily expressed in podocytes in glomeruli, whereas their functional receptors Flk-1 and Tie2, respectively, have been found on glomerular endothelium, suggesting a regulatory role of podocytes in inducing and maintaining glomerular capillary structure $(2,3)$. Under disease conditions, VEGF and Ang-1 direct the protection against, and/or the repair from, injury to glomerular endothelium $(4-6)$.

In vivo studies of glomerular development in zebrafish mutants revealed that maturation of podocytes occurred slightly earlier than maturation of glomerular capillary loops

Received January 2, 2007; accepted June 3, 2007.

Correspondence: Ji Ma, M.D., Ph.D., C4204 MCN, 1161 21st Avenue South, Vanderbilt University Medical Center, Nashville, TN 37232; e-mail: ji.ma@vanderbilt.edu

This work was supported by the National Institutes of Health grants DK-44757 and DK-37868, the Ministry of Education of China grant 81038 and the National Natural Science Foundation of China grant 30200125. and was independent of endothelial cells (7). Moreover, podocytes appeared to play an important role in guiding glomerular capillary formation (8). Numbers of both mature and immature glomeruli were reduced by administration of VEGF antibody to neonatal mice, and some glomeruli lacked capillary tufts (9). Recently, malformation of glomerular capillary loops was reported in mice with either podocyte-specific deficiency of VEGF-A or overexpression of podocyte VEGF164 (10), indicating a key regulatory role of podocyte-derived VEGF in maturation of glomerular capillaries. In the present study, we investigated whether injury of podocytes affects maturation of glomerular capillaries in developing glomeruli. Given that puromycin aminonucleoside (PAN) has been used as a podocyte toxin in a variety of experiment settings in adult rats as well as in a differentiated cultured mouse podocyte cell line (11), we treated neonatal mice with PAN to investigate effects of podocyte injury on different stages of glomerular development.

\section{METHODS}

Animal models. ICR neonatal mice (Harlan, Indianapolis, IN) were randomly assigned to receive $0.1 \mathrm{mg} / \mathrm{g}$ body weight of PAN (Sigma Chemical Co., St. Louis, MO) or an equal volume of normal saline (NS) intraperitoneally at 1 day postpartum (dpp). The neonates were weaned at $21 \mathrm{dpp}$. All animals were housed in a standard pathogen-free facility with 12-h light/dark cycle. All animal procedures used in the study were approved by Institutional Animal Care and Use Committee (IACUC) at Vanderbilt University.

The experimental animals were killed at 2, 4, 8, 12, 30, 60, and $90 \mathrm{dpp}$, respectively. Mouse urine was collected from the bladder upon sacrifice at 4 , 8, and $12 \mathrm{dpp}$ and from metabolic cages (Nalgene, Rochester, NY) for $24 \mathrm{~h}$ at 30 and $60 \mathrm{dpp}$. Urinary excretion of protein was examined by gradient sodium dodecyl sulfate-polyacrylamide gel electrophoresis. Systolic blood pressure was measured by tail-cuff impedance plethysmography (Visitech BP-2000, Apex, NC) at 60 and 90 dpp.

Kidney tissue was weighed at sacrifice and harvested for morphologic studies. A portion of renal cortex was snap-frozen in liquid nitrogen for mRNA analyses.

Morphology and immunohistochemistry. A longitudinal section of the kidney cut through the papilla was fixed in $4 \%$ buffered paraformaldehyde and then processed and embedded in paraffin for histologic evaluation. Paraffinembedded kidney sections were cut at $3-\mu \mathrm{m}$ thickness. Glomerular maturation index (GMI, 0-3 scale) at 12 and $30 \mathrm{dpp}$ was determined on periodic acid-Schiff (PAS)-stained sections as previously described (12).

A piece of kidney cortex was routinely processed for examination with transmission electron microscopy (EM). EM study was performed on three animals from each group at 2, 4, 8, and $30 \mathrm{dpp}$. About six glomeruli were examined by EM for each animal.

Abbreviations: Ang-1, angiopoietin-1; dpp, days postpartum; EM, electron microscopy; GBM, glomerular basement membrane; KW, kidney weight; NS, normal saline; PAN, puromycin aminonucleoside; VEGF, vascular endothelial growth factor; WT-1, Wilms tumor suppressor gene 1 
Table 1. Primers used for real-time PCR

\begin{tabular}{|c|c|c|c|}
\hline Gene & Forward primer & Reverse primer & Reference \\
\hline GAPDH & 5'-TGG ACC ACC AAC TGC TTA G-3' & 5'-GGA TGC AGG GAT GAT GTT C-3' & 14 \\
\hline VEGF164 & 5'-AAC GAT GAA GCC CTG GAG TG-3' & 5'-GAC AAA CAA ATG CTT TCT CCG-3' & 14 \\
\hline Flk-1 & $5^{\prime}$-AGG GTT TCT GCT GGG TGG A-3' & 5'-TGA GCC TTT ACC GCC ACT TG-3' & 14 \\
\hline Ang-2 & 5'-AGC AGA TTT TGG ATC AGA CCA G-3' & 5'-GCT CCT TCA TGG ACT GTA GCT G-3' & 15 \\
\hline Tie1 & $5^{\prime}$-TGG AGT CCA CTG TGA AAA GTC A-3' & 5'-GCA TCG TCC CTA TGT TGA AC TC-3' & 15 \\
\hline Tie2 & 5'-CGG CTT AGT TCT CTG TGG AGT C-3' & 5'-GGC ATC AGA CAC AAG AGG TAG G-3' & 15 \\
\hline Integrin $\beta 1$ & $5^{\prime}$-TGG TCC ATG TCT AGC GTC A-3' & $5^{\prime}$-TCC TGT GCA CAC GTG TCT T-3' & \\
\hline
\end{tabular}

Wilms tumor suppressor gene 1 (WT-1), a podocyte marker; CD31, an endothelial marker; and VEGF were detected by immunohistochemistry. Briefly, after quenching endogenous peroxidase with $3 \% \mathrm{H}_{2} \mathrm{O}_{2}$ in methanol, tissue sections were microwaved for WT-1 staining or treated with $0.2 \mathrm{mg} / \mathrm{mL}$ trypsin/phosphate-buffered saline at room temperature for CD31 or VEGF staining. Sections were then incubated with rabbit anti-mouse WT-1 (Santa Cruz, Santa Cruz, CA) at 1:800 dilution or rat anti-mouse CD31 (BD Pharmingen, San Diego, CA) at 1:100 dilution, or goat anti-mouse VEGF (Sigma Chemical Co.) at 1:50 dilution at $4^{\circ} \mathrm{C}$ overnight. Standard blocking, secondary antibody incubation, and developing procedures were applied according to the instructions provided by the corresponding $\mathrm{ABC}$ kits (Vectorlabs, Burlingame, CA). Immunostaining was visualized by diaminobenzidine.

After immunohistochemistry, morphometric analysis was performed under a $40 \times$ objective on at least 30 consecutive glomeruli in the cortex, with the average calculated for each animal. Cells with positive WT-1 staining within the glomerulus were counted and averaged. Glomerular cells located outside the GBM with positive VEGF staining were counted. CD31+ area and glomerular area were measured using KS300 software (Kontron Electronic $\mathrm{GmbH}$, Eching, Germany). Relative glomerular capillary area for each glomerulus was calculated by the ratio of the glomerular CD31+ area to the glomerular area. All morphometric analyses were done by trained laboratory staff who were blinded to group allocation.

Quantitative real-time polymerase chain reaction (PCR). Total RNA was extracted using RNeasy Mini Kit (Qiagen, Valencia, CA). Reverse transcription was performed using TaqMan Reverse Transcription Kit (Applied Biosystems, Branchburg, NJ) in a total reaction volume of $40 \mu \mathrm{L}$ with $2 \mu \mathrm{g}$ total RNA. Quantitative real-time PCR was performed in a total reaction volume of $25 \mu \mathrm{L}$ using iQ SYBR Green Supermix (Bio-Rad, Hercules, CA) and $0.5 \mu \mathrm{L}$ cDNA. The reaction was carried out using the iCycler iQ Multi-Color Real Time PCR Detection System (Bio-Rad) under the following conditions: polymerase activation for $3 \mathrm{~min}$ at $95^{\circ} \mathrm{C}$ and amplification for 40 cycles of $20 \mathrm{~s}$ at $95^{\circ} \mathrm{C}, 20 \mathrm{~s}$ at $60^{\circ} \mathrm{C}$, and $40 \mathrm{~s}$ at $72^{\circ} \mathrm{C}$. After amplification, a final melting curve was obtained for each sample by cooling the reaction mixture to $55^{\circ} \mathrm{C}$ and then slowly heating it to $95^{\circ} \mathrm{C}$ at $0.2^{\circ} \mathrm{C} / \mathrm{s}$. Experimental cycle threshold $(\mathrm{Ct})$ values were normalized to glyceraldehyde-3-phosphate dehydrogenase (GAPDH) measured on the same plate, and the relative levels of gene expression were determined by using the $2^{-\Delta \Delta \mathrm{Ct}}$ method (13). Primers used to detect the expression levels of different genes are listed in Table 1 (14-16).

Statistical analysis. Results are expressed as mean \pm SD in the text and tables and mean \pm SEM in figures. Analysis of variance (ANOVA) and a post hoc Scheffé test were used to evaluate differences between NS- and PAN- injected animals. The results were further confirmed by nonparametric MannWhitney $U$ test. All tests were two tailed, and $p<0.05$ was considered statistically significant.

\section{RESULTS}

Body and kidney weights. At baseline at $1 \mathrm{dpp}$, the body weight of ICR neonates ranged from 1.7 to $2.3 \mathrm{~g}$, which was not significantly different between male and female pups or between the pups allocated to PAN versus NS injection. The percentages of male versus female in the PAN group versus NS group were not significantly different by $\chi^{2}$ test (data not shown) at any time point. There was no noted gender difference in histologic examinations. Therefore, data from different genders were combined in this study. PAN-injected mice had significantly lower BW and KW compared with NS controls at 4, 8, 12, 30, 60, and $90 \mathrm{dpp}$, and lower KW/BW ratio at 4, 8, and 12 dpp (Table 2).

Morphology. PAN mice had smaller glomerular tufts and more single branches with normal size of Bowman's capsule thus enlarged Bowman's space in about $5 \%-6 \%$ of superficial glomeruli versus NS mice, indicating arrested development of these glomerular capillary loops (Fig. $1 B, C, E, F$ ). The percentage of those abnormal glomeruli was decreased, and the remaining abnormal glomeruli were smaller in PAN mice at $30 \mathrm{dpp}$ (Fig. 1H, I). Abnormal glomeruli were not detected by $90 \mathrm{dpp}$ (Fig. $1 K, L$ ). Thus, glomeruli with underdeveloped capillary tuft appeared to have been gradually absorbed. The glomerular maturation index was significantly decreased in superficial glomeruli at 12 and $30 \mathrm{dpp}$ in PAN versus NS mice (Table 3). The density of glomeruli at $12 \mathrm{dpp}$ was increased from $25.11 \pm 1.92$ glomeruli $/ \mathrm{mm}^{2}$ cortical area in NS-injected kidneys (Fig. $1 D$ ) to $36.31 \pm 3.96$ glomeruli $/ \mathrm{mm}^{2}$ cortical

Table 2. Changes in $B W$ and $K W$ in neonates after PAN injection

\begin{tabular}{|c|c|c|c|c|c|c|c|c|}
\hline & \multicolumn{2}{|c|}{$\mathrm{N}$} & \multicolumn{2}{|c|}{ BW (g) } & \multicolumn{2}{|c|}{ KW (mg) } & \multicolumn{2}{|c|}{ KW/BW (mg/g) } \\
\hline $4 \mathrm{dpp}$ & 6 & 6 & $3.13 \pm 0.19$ & $2.76 \pm 0.31 *$ & $35.0 \pm 1.6$ & $28.8 \pm 4.4^{*}$ & $11.4 \pm 0.8$ & $10.4 \pm 0.8^{*}$ \\
\hline $12 \mathrm{dpp}$ & 5 & 7 & $7.48 \pm 0.55$ & $4.47 \pm 1.16^{*}$ & $126.1 \pm 10.8$ & $60.4 \pm 15.8^{* *}$ & $16.8 \pm 0.4$ & $13.5 \pm 1.4^{*}$ \\
\hline $30 \mathrm{dpp}$ & 5 & 7 & $23.01 \pm 2.43$ & $14.88 \pm 5.84 * *$ & $422.6 \pm 78.3$ & $252.6 \pm 115.2^{* *}$ & $18.4 \pm 2.4$ & $15.7 \pm 4.2$ \\
\hline $60 \mathrm{dpp}$ & 6 & 7 & $36.13 \pm 2.47$ & $25.96 \pm 4.50 * *$ & $680.2 \pm 124.0$ & $437.8 \pm 123.9^{*}$ & $18.7 \pm 2.4$ & $16.8 \pm 3.4$ \\
\hline
\end{tabular}

$* p<0.05$.

** $p<0.01$ vs NS at the same time point. 

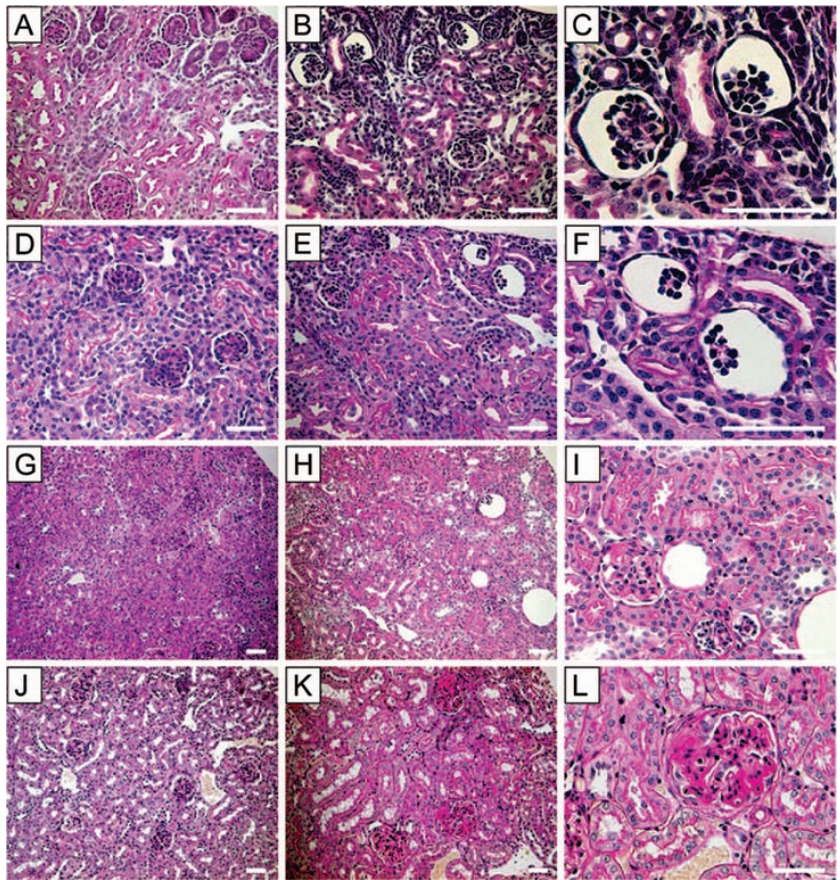

Figure 1. Histologic changes of neonatal kidneys after injection with PAN. After PAN injection at $1 \mathrm{dpp}$, superficial glomeruli showed smaller glomerular tufts with less maturation and simpler branches at $4 \mathrm{dpp}(B), 12 \mathrm{dpp}(E)$, and $30 \mathrm{dpp}(H)$ when compared with their littermates injected with NS at 4 dpp (A), $12 \mathrm{dpp}(D)$, and $30 \mathrm{dpp}(G)$, respectively. Glomerular mesangial expansion and segmental glomerulosclerosis were noted in PAN-injected kidneys at $60 \mathrm{dpp}$ and $90 \mathrm{dpp}(K)$. (J) With NS injection at $90 \mathrm{dpp} . C, \mathrm{~F}, I$, $L$ enlarged from $B, E, H, K$, respectively. Scale bars $=50 \mu \mathrm{m}$ (periodic acid-Schiff).

area by PAN injection (Fig. 1E), which along with decreased $\mathrm{KW}$, suggests less tubular development. Along with occasional proteinaceous casts, marked glomerular mesangial expansion and hypercellularity and glomerulosclerosis were detected in $34.2 \pm 7.1 \%$ of both superficial and deep glomeruli in PAN-injected kidneys at $60 \mathrm{dpp}$ and in $51.9 \pm 7.9 \%$ of glomeruli at $90 \mathrm{dpp}$ (Fig. $1 K, L$; each $n=7$ ), lesions that were not observed in NS kidneys (each time point $n=6$ ).

EM revealed $80 \%$ to nearly complete podocyte foot process effacement, segmental microvillous transformation, and lucency of the lamina rara interna of the GBM with intact endothelium in superficial glomeruli as early as $1 \mathrm{~d}$ after PAN injection (Fig. 2B), whereas NS-injected kidney showed wellformed foot processes and normal GBM (Fig. 1A). These findings were still prominent at $8 \mathrm{dpp}$ (Fig. 2C). Foot processes were restored at $30 \mathrm{dpp}$ in PAN-injected mice, accompanied by increased mesangial matrix and cellularity (Fig. 2D).

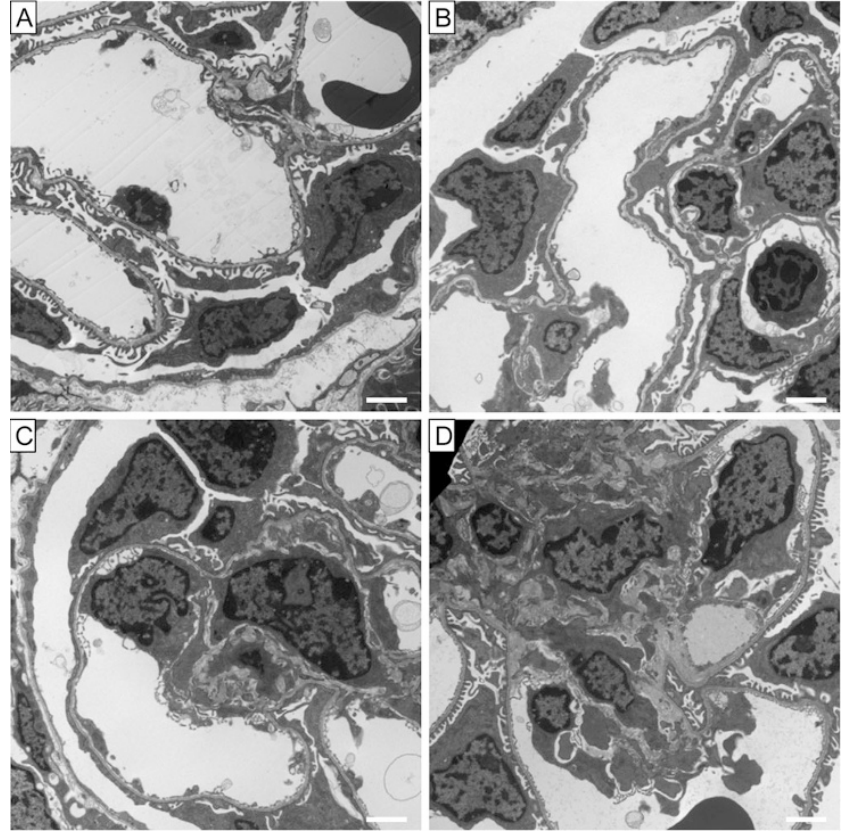

Figure 2. Changes of neonatal kidneys by EM. Normal neonatal glomeruli showed well-formed podocyte foot processes and normal GBM at $2 \mathrm{dpp}(A)$. In contrast, injection with PAN induced foot process effacement, microvillous transformation, and lucency of the lamina rara interna as early as $2 \mathrm{dpp}(B)$, lesions that were still prominent at $8 \mathrm{dpp}(C)$. At $30 \mathrm{dpp}$, PAN-injected glomeruli showed increased mesangial matrix and cellularity with restored foot processes $(D)$. Scale bars $=2 \mu \mathrm{m}$.

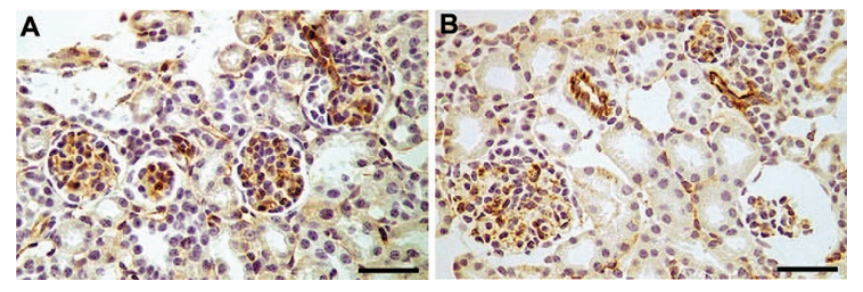

Figure 3. CD 31 (PECAM-1) immunostaining at $12 \mathrm{dpp}$ after injection with PAN. PAN injection induced significantly decreased area of glomerular endothelium at $12 \mathrm{dpp}(B)$ compared with injection with NS $(A)$. Scale bars = $50 \mu \mathrm{m}$.

PAN-injected kidney showed significantly decreased relative glomerular capillary volume assessed as CD31+ area (PAN $0.047 \pm 0.022$ versus NS $0.127 \pm 0.041, n=5, p<$ 0.01 ) at $12 \mathrm{dpp}$ (Fig. 3). There was no significant difference in the number of VEGF- or WT-1-positive podocytes per glomerulus in PAN- versus NS-treated kidneys at 2, 4, or $12 \mathrm{dpp}$ (Table 4).

Animal survival, systolic blood pressure, and proteinuria. Most of the PAN-injected mice died by 3 mo, whereas NS injection did not cause any spontaneous deaths during the

Table 3. Glomerular maturation index

\begin{tabular}{|c|c|c|c|c|c|c|c|c|}
\hline & \multicolumn{2}{|c|}{$\mathrm{N}$} & \multicolumn{2}{|c|}{ Superficial glomeruli } & \multicolumn{2}{|c|}{ Deep glomeruli } & \multicolumn{2}{|c|}{ All glomeruli } \\
\hline $12 \mathrm{dpp}$ & 5 & 7 & $2.47 \pm 0.11$ & $2.04 \pm 0.18^{*}$ & $2.96 \pm 0.04$ & $2.92 \pm 0.09$ & $2.55 \pm 0.10$ & $2.23 \pm 015^{*}$ \\
\hline
\end{tabular}

\footnotetext{
$* p<0.05$ vs NS at the same time point.
} 
Table 4. WT-1- and or VEGF-positive podocytes in neonatal glomeruli

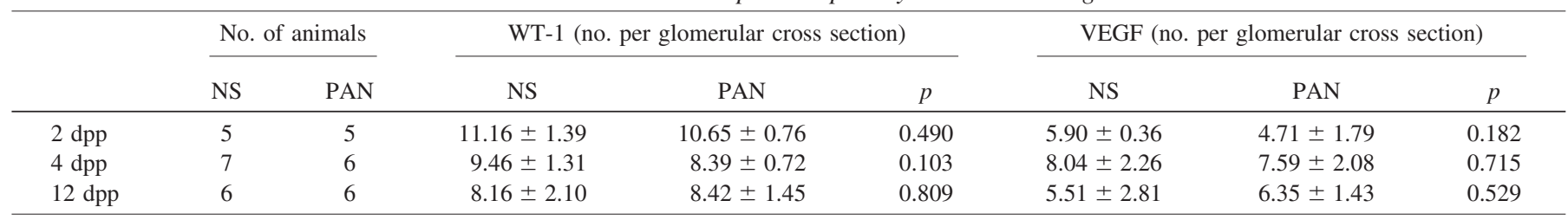

study period. At $60 \mathrm{dpp}$, PAN mice showed significantly increased systolic blood pressure compared with the NS controls (PAN $133.4 \pm 28.7 \mathrm{~mm} \mathrm{Hg}$ versus NS $105.5 \pm 9.1 \mathrm{~mm}$ $\mathrm{Hg}, p<0.05, n=8$ for PAN and $n=7$ for NS). Proteinuria was not present in any animals at 4,8 , or $12 \mathrm{dpp}$. Starting from $60 \mathrm{dpp}$, mild proteinuria was detected in PAN-injected mice but not in NS mice.

Results of mRNA expression examined by quantitative real-time PCR. PAN injection induced significantly decreased mRNA expression in Flk-1 and Tie2, increased expression in Ang-1, with no changes in VEGF164 at 2 dpp compared with NS (Fig. 4). We also examined the expression of synaptopodin, a podocyte foot process structural protein, and $\alpha 3 \beta 1$ integrin, a protein mediating attachment of podocytes to the GBM as well as signal transduction between podocytes and GBM (17). There was no significant difference in mRNA expression of synaptopodin or $\alpha 3 \beta 1$-integrin between PANand NS-injected renal cortex at $2 \mathrm{dpp}$.

\section{DISCUSSION}

The glomerular podocyte has been regarded as one of the essential components of the glomerular filtration barrier, regulating the filtration function of glomeruli. Recent in vivo studies with mutant zebrafish (8) and mice (10) suggest a regulatory role of podocytes in directing maturation of glomerular capillaries. In the present study, we found that podocytes of neonatal ICR mice are susceptible to PAN, resulting in extensive podocyte foot process effacement, up-regulated expression of Ang-1 and down-regulation of Flk-1 and Tie2, and abnormal development of glomerular capillaries in the least differentiated superficial glomeruli. These changes led to proteinuria, hypertension, glomerular mesangial expansion, and focal segmental sclerosis in the adult stage.

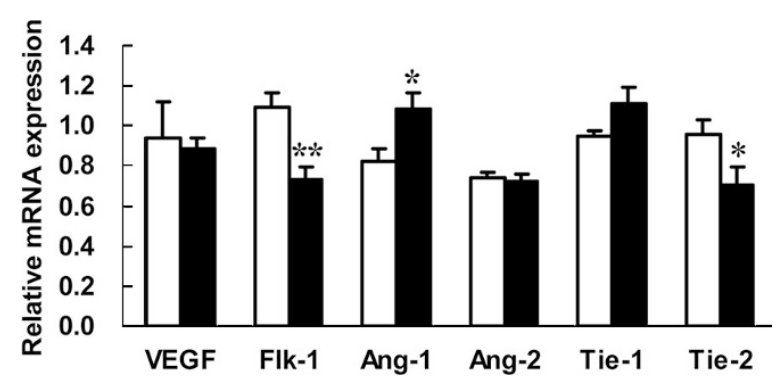

Figure 4. Renal cortical mRNA expression examined by quantitative realtime PCR. Compared with control mice injected with NS (open columns), injection with PAN (closed columns) induced significantly decreased mRNA expression in Flk-1 and Tie2, increased expression in Ang-1, without changes in VEGF164 at 2 dpp. *p $<0.05 ; * * p<0.01$ vs NS. For each group, duplicate samples from each of four individual animals were examined.
Podocyte damage has been regarded as a major culprit contributing to the progression of glomerulosclerosis (18). Podocytes are terminally differentiated cells with limited capability to regenerate or proliferate in mature glomeruli (19). However, study of podocytes during the developing stage could shed light on the mechanism of glomerulosclerosis. Rodent kidneys are still maturing at birth, with less mature superficial versus deep cortical glomeruli. The maturation of glomeruli of rodents is not complete until $3 \mathrm{wk}$ after birth (20). In mature rats, PAN injection has been used to induce podocyte injury and massive proteinuria (21). Multiple injections of PAN can lead to irreversible podocyte damage, nephrotic syndrome, and later glomerulosclerosis $(22,23)$. Adult mice do not share this susceptibility (24). However, a differentiated podocyte cell line of mouse origin is susceptible to PAN (11). In our study, even though $100 \mathrm{mg} / \mathrm{kg}$ body weight of PAN did not induce podocyte injury or proteinuria in adult ICR mice (data not shown), this dose of PAN induced prompt changes in podocytes in neonatal mouse kidneys. Thus, in this study, we have established a new in vivo method to induce podocyte injury in neonatal mice.

In our study, we found that PAN-induced podocyte injury resulted in arrest of glomerular capillary development, shown by a significantly decreased glomerular maturation index and decreased positive staining for the endothelial marker CD31. VEGF164 mRNA and protein assessed by real-time PCR and immunohistochemistry, respectively, were not affected. However, renal Ang-1 gene expression was significantly upregulated at $1 \mathrm{~d}$ after PAN injection, suggesting that an imbalance between Ang-1 and VEGF may underlie the abnormal development of glomerular capillaries in puromycininduced neonatal nephropathy in ICR mice. Down-regulation of the VEGF receptor Flk-1 and Ang-1 receptor Tie2, which may be attributed to the attenuated endothelial maturation after PAN injection, may also play a role in the malformation of glomerular capillaries found in this study.

Both human and animal studies have shown that loss and/or injury of podocytes are related to proteinuria and glomerulosclerosis in various disease states including primary glomerulopathies, aging, hypertension, and diabetes mellitus (25-28). PAN injection in neonatal mice caused arrested development of capillary loops in some glomeruli, with global absorption after 1 mo of age. In view of prevailing notions, the subsequent proteinuria, hypertension, and glomerulosclerosis could be a consequence of a reduced number of nephrons in early life.

Even though EM showed nearly complete foot process effacement in all examined glomeruli at an early stage, there was no trace of proteinuria during this period. This lack of 
proteinuria was paralleled by normal expression of synaptopodin, $\alpha 3 \beta 1$-integrin, and VEGF. Further studies on podocyte slit diaphragm proteins and cytoskeleton molecules may elucidate mechanisms underlying the dissociation between foot process effacement and proteinuria in neonatal PAN-induced nephropathy.

Of note, PAN injection in neonatal mice also induced growth retardation, which may not be exclusive from the pathogenesis of attenuated nephron development found in this study. Studies with specific podocyte injury models during glomerular development will be helpful to further elucidate the role of podocytes in maturation of glomerular capillaries.

In summary, our study has established that maturing podocytes are susceptible to PAN in mice. This mild podocyte injury in maturing nephrons causes developmental abnormality in glomerular capillaries and subsequent glomerulosclerosis. Imbalance of VEGF and angiopoietin systems may be involved in the pathogenesis of this neonatal PAN nephropathy. Further studies on the interactions between podocytes and glomerular endothelium in early life may shed light on the mechanisms of sclerosis and proteinuria in adults.

Acknowledgments. The authors thank Xiaofeng Fan for excellent technical assistance.

\section{REFERENCES}

1. Camici M 2005 Renal glomerular permselectivity and vascular endothelium. Biomed Pharmacother 59:30-37

2. Simon M, Grone HJ, Johren O, Kullmer J, Plate KH, Risau W, Fuchs E 1995 Expression of vascular endothelial growth factor and its receptors in human renal ontogenesis and in adult kidney. Am J Physiol 268:F240-F250

3. Satchell SC, Harper SJ, Tooke JE, Kerjaschki D, Saleem MA, Mathieson PW 2002 Human podocytes express angiopoietin 1, a potential regulator of glomerular vascular endothelial growth factor. J Am Soc Nephrol 13:544-550

4. Takazawa Y, Maeshima Y, Kitayama H, Yamamoto Y, Kawachi H, Shimizu F, Matsui H, Sugiyama H, Yamasaki Y, Makino H 2005 Infusion of angiotensin II reduces loss of glomerular capillary area in the early phase of anti-Thy-1.1 nephritis possibly via regulating angiogenesis-associated factors. Kidney Int 68:704-722

5. Masuda Y, Shimizu A, Mori T, Ishiwata T, Kitamura H, Ohashi R, Ishizaki M, Asano G, Sugisaki Y, Yamanaka N 2001 Vascular endothelial growth factor enhances glomerular capillary repair and accelerates resolution of experimentally induced glomerulonephritis. Am J Pathol 159:599-608

6. Yuan HT, Tipping PG, Li XZ, Long DA, Woolf AS 2002 Angiopoietin correlates with glomerular capillary loss in anti-glomerular basement membrane glomerulonephritis. Kidney Int 61:2078-2089

7. Majumdar A, Drummond IA 1999 Podocyte differentiation in the absence of endothelial cells as revealed in the zebrafish avascular mutant, cloche. Dev Genet 24:220-229
8. Majumdar A, Drummond IA 2000 The zebrafish floating head mutant demonstrates podocytes play an important role in directing glomerular differentiation. Dev Biol 222:147-157

9. Kitamoto Y, Tokunaga H, Tomita K 1997 Vascular endothelial growth factor is an essential molecule for mouse kidney development: glomerulogenesis and nephrogenesis. J Clin Invest 99:2351-2357

10. Eremina V, Sood M, Haigh J, Nagy A, Lajoie G, Ferrara N, Gerber HP, Kikkawa Y, Miner JH, Quaggin SE 2003 Glomerular-specific alterations of VEGF-A expression lead to distinct congenital and acquired renal diseases. J Clin Invest 111:707-716

11. Vega-Warner V, Ransom RF, Vincent AM, Brosius FC, Smoyer WE 2004 Induction of antioxidant enzymes in murine podocytes precedes injury by puromycin aminonucleoside. Kidney Int 66:1881-1889

12. Niimura F, Labosky PA, Kakuchi J, Okubo S, Yoshida H, Oikawa T, Ichiki T, Naftilan AJ, Fogo A, Inagami T, Hogan BL, Ichikawa I 1995 Gene targeting in mice reveals a requirement for angiotensin in the development and maintenance of kidney morphology and growth factor regulation. J Clin Invest 96:2947-2954

13. Livak KJ, Schmittgen TD 2001 Analysis of relative gene expression data using real-time quantitative PCR and the 2(-Delta Delta C(T)) method. Methods 25:402408

14. Ploplis VA, Balsara R, Sandoval-Cooper MJ, Yin ZJ, Batten J, Modi N, Gadoua D, Donahue D, Martin JA, Castellino FJ 2004 Enhanced in vitro proliferation of aortic endothelial cells from plasminogen activator inhibitor-1-deficient mice. J Biol Chem 279:6143-6151

15. Zhang ZG, Zhang L, Tsang W, Soltanian-Zadeh H, Morris D, Zhang R, Goussev A, Powers C, Yeich T, Chopp M 2002 Correlation of VEGF and angiopoietin expression with disruption of blood-brain barrier and angiogenesis after focal cerebral ischemia. J Cereb Blood Flow Metab 22:379-392

16. Roselli S, Heidet L, Sich M, Henger A, Kretzler M, Gubler MC, Antignac C 2004 Early glomerular filtration defect and severe renal disease in podocin-deficient mice. Mol Cell Biol 24:550-560

17. Adler S 1992 Characterization of glomerular epithelial cell matrix receptors. Am J Pathol 141:571-578

18. Kriz W 2002 Podocyte is the major culprit accounting for the progression of chronic renal disease. Microsc Res Tech 57:189-195

19. Sasaki T, Osawa G 1993 A kinetic study of the glomerular cells of developing and mature rat kidneys using an anti-bromodeoxyuridine monoclonal antibody. Nippon Jinzo Gakkai Shi 35:1213-1219

20. Hyink DP, Abrahamson DR 1995 Origin of the glomerular vasculature in the developing kidney. Semin Nephrol 15:300-314

21. Pinto JA, Brewer DB 1975 Combined light and electron-microscope morphometric studies of acute puromycin aminonucleoside nephropathy in rats. J Pathol 116:149164

22. Glasser RJ, Velosa JA, Michael AF 1977 Experimental model of focal sclerosis. I Relationship to protein excretion in aminonucleoside nephrosis. Lab Invest 36:519526

23. Fogo AB 2003 Animal models of FSGS: lessons for pathogenesis and treatment Semin Nephrol 23:161-171

24. Wang L, Fields TA, Pazmino K, Dai Q, Burchette JL, Howell DN, Coffman TM, Spurney RF 2005 Activation of G\{alpha\}q-coupled signaling pathways in glomerular podocytes promotes renal injury. J Am Soc Nephrol 16:3611-3622

25. Macconi D, Bonomelli M, Benigni A, Plati T, Sangalli F, Longaretti L, Conti S, Kawachi H, Hill P, Remuzzi G, Remuzzi A 2006 Pathophysiologic implications of reduced podocyte number in a rat model of progressive glomerular injury. Am J Pathol 168:42-54

26. Wolf G, Chen S, Ziyadeh FN 2005 From the periphery of the glomerular capillary wall toward the center of disease: podocyte injury comes of age in diabetic nephropathy. Diabetes 54:1626-1634

27. Yu D, Petermann A, Kunter U, Rong S, Shankland SJ, Floege J 2005 Urinary podocyte loss is a more specific marker of ongoing glomerular damage than proteinuria. J Am Soc Nephrol 16:1733-1741

28. Ortmann J, Amann K, Brandes RP, Kretzler M, Munter K, Parekh N, Traupe T, Lange M, Lattmann T, Barton M 2004 Role of podocytes for reversal of glomerulosclerosis and proteinuria in the aging kidney after endothelin inhibition. Hypertension 44:974-981 